\title{
Technological and Physics Assessments on Heating and Current Drive Systems for DEMO
}

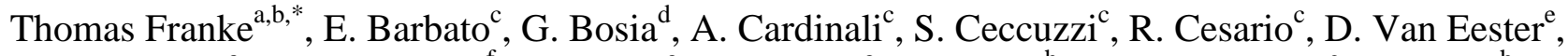
G. Federici ${ }^{\mathrm{a}}$, G. Gantenbein ${ }^{\mathrm{f}}$, W. Helou ${ }^{\mathrm{g}}$, J. Hillairet ${ }^{\mathrm{g}}$, I. Jenkins ${ }^{\mathrm{h}}$, Ye. O. Kazakov ${ }^{\mathrm{e}}$, R. Kemp ${ }^{\mathrm{h}}$, E. Lerche ${ }^{\mathrm{e}}$, F. Mirizzi ${ }^{\mathrm{c}}$, J.-M. Noterdaeme ${ }^{\mathrm{b}}$, E. Poli ${ }^{\mathrm{b}}$, L. Porte ${ }^{\mathrm{i}}$, G. L. Ravera ${ }^{\mathrm{c}}$, E. Surrey ${ }^{\mathrm{h}}$, G. Tardini $^{\mathrm{b}}$, M. Q. Tran ${ }^{\mathrm{i}}$, C. Tsironis ${ }^{\mathrm{j}, \mathrm{k}}$, A. A. Tuccillo ${ }^{\mathrm{c}}$, R. Wenninger ${ }^{\mathrm{a}}$, H. Zohm $^{\mathrm{b}}$ and the EFDA PPP\&T team ${ }^{\mathrm{a}}$

${ }^{a}$ EFDA Close Support Unit, Boltzmannstr. 2, D-85748 Garching, Germany

${ }^{b}$ Max Planck Institute for Plasma Physics, Boltzmannstr. 2, D-85748 Garching, Germany

${ }^{c}$ Unità Tecnica Fusione ENEA, C. R. Frascati, via E. Fermi 45, 00044 Frascati (Roma), Italy

${ }^{d}$ Department of Physics, University of Turin, Via P. Giuria 1, 10125 Turin, Italy

${ }^{e}$ Laboratory for Plasma Physics, LPP-ERM/KMS, TEC \& Belgian EUROfusion Consortium partner, Brussels, Belgium

${ }^{f}$ Karlsruhe Institute of Technology (KIT), Association EURATOM-KIT, Kaiserstrasse 12, 76131 Karlsruhe, Germany

${ }^{g}$ CEA, IRFM, F-13108 Saint-Paul-lez-Durance, France

${ }^{h}$ CCFE, Culham Science Centre, Abingdon, OX14 3DB, UK

${ }^{i}$ Centre de Recherches en Physique des Plasmas, Ecole Polytechnique Fédérale de Lausanne (EPFL), Station13, CH1015, Lausanne, Switzerland

${ }^{j}$ Department of Physics, Aristotle University of Thessaloniki, Greece

${ }^{k}$ School of Electrical and Computer Engineering, National Technical University of Athens, Greece

The physics requirements of the heating and current (H\&CD) systems in a Demonstration Fusion Power Plant (DEMO) are often beyond the actual level of design maturity and technology readiness required. The recent EU fusion roadmap advocates a pragmatic approach and favours, for the initial design integration studies, systems to be as much as possible, extrapolated from the ITER experience. To reach the goal of demonstrating the production of electricity in DEMO with a closed fuel cycle by 2050, one must ensure reliability, availability, maintainability, inspectability (RAMI) as well as performance, efficiency and optimized design for the H\&CD systems.

In the recent Power Plant Physics \& Technology (PPP\&T) Work Programme a number of H\&CD studies were performed. The four H\&CD systems Neutral Beam (NB) Injection, Electron Cyclotron (EC), Ion Cyclotron (IC) and Lower Hybrid (LH) were considered. First, a physics optimisation study was made assuming all technologies are available and identifying which parameters are needed to optimize performance for given plasma parameters. Separately, the (i) technological maturity was considered (e.g. $240 \mathrm{GHz}$ gyrotrons for EC) and (ii) technologies were adapted (e.g. multi-stage depressed collector for EC) or (iii) novel solutions (e.g. photo-neutralization for NB or new antennae concepts for IC) were studied to overcome the limitations of the present H\&CD systems with respect to DEMO requirements. Further constraints imposed by remote maintenance or breeding blanket interactions were considered.

Keywords: DEMO, Heating \& Current Drive, Neutral Beam, Electron Cyclotron, Ion Cyclotron, Lower Hybrid

\section{Foreword}

In this paper the extensive work of the various H\&CD groups during the last years will be highlighted and some of the important new findings summarized. More details can be found in the reference list [1]-[19]. All final reports of the work-programmes 2011-2013 are available in the EFDA IDM database for the EUROfusion Consortium Members and Auditors [20].

\section{Introduction}

To prepare the DEMO conceptual design activity (CDA) phase initiated in 2014, work was carried out in a pre-conceptual design phase during 2011-2013 in view of the engineering design activity (EDA) phase of DEMO starting around 2020 [1] and chasing the Fusion Roadmap [2] target operating the prototype fusion power plant in 2050. The work on H\&CD systems was based on two regimes of plasma operations which are (i) a pulsed machine with either no (or marginal) current drive (CD) power or with reasonable but limited bulk $\mathrm{CD}$ power in case of an extended long pulse machine and (ii) a full steady-state machine with significant H\&CD power. The work described here concentrates on the case of a pulsed machine [2]. Full steady state operation requires challenging, but not unrealistic efficient technologies [2] compared to current state-of-the-art of $\mathrm{H} \& \mathrm{CD}$ systems regarding wall-plug and CD efficiencies. The recirculating power is limited such that the net electric power to the grid is $\sim 500 \mathrm{MW}_{\mathrm{e}}$ and the pulse flat top length is optimized to achieve a high duty cycle [4].

\section{Power Plant Description}

\subsection{DEMO parameters}

The basic tokamak parameters of the current pulsed DEMO reference design are given in Table 1 and derived from PROCESS code [10]. This 0D-code is taking into account the main DEMO fusion power plant systems (e.g. pumping, fuelling, magnetic coils, balance 
of plant and site) to optimize the tokamak and its performance and costs.

Table 1. Summary of a DEMO PROCESS code results [2].

\begin{tabular}{|l|l|}
\hline Plasma Fusion Power $\left(P_{\text {fusion }}\right)$ & $1572 \mathrm{MW}$ \\
\hline Thermal Power & $1972 \mathrm{MW}$ \\
\hline Radiation Losses $\left(P_{\text {rad }}\right)$ & $186 \mathrm{MW}$ \\
\hline$P_{\text {sep }}, P_{\text {sep }} / \mathrm{R}$ & $180 \mathrm{MW}, 20 \mathrm{MW} / \mathrm{m}$ \\
\hline$P_{\mathrm{L}-\mathrm{H}}$ & $136 \mathrm{MW}$ \\
\hline Plasma Current $\left(I_{\mathrm{P}}\right)$ & $14 \mathrm{MA}$ \\
\hline Thermal Efficiency & $45 \%$ \\
\hline Electricity Output & $500 \mathrm{MW}$ \\
\hline Major Radius & $9.0 \mathrm{~m}$ \\
\hline Minor Radius & $2.25 \mathrm{~m}$ \\
\hline Aspect Ratio & 4 \\
\hline Toroidal Field (TF) Strength & $6.8 \mathrm{~T}$ \\
\hline Number of TF coils & 16 \\
\hline Neutral Beam Energy & $1 \mathrm{MeV}$ \\
\hline$Z_{\text {eff }}$ & 1.98 \\
\hline Auxiliary Heating Power $\left(P_{\text {aux }}\right)$ & $50 \mathrm{MW}$ \\
\hline Pulse Length & $\sim 2.3 \mathrm{~h}$ \\
\hline
\end{tabular}

To improve the DEMO tokamak, in terms of plasma control (vertical stability), disruption forces and a number of other issues, an aspect-ratio trade-off study is under way [5]. The study includes the choice of a different EC frequency for each chosen magnetic field strength and the NB layout (e.g. tangential angle). The number of the TF coils is varied to see influences to the magnetic ripple and therefore the ion losses.

\subsection{H\&CD Power Requirements}

The H\&CD power is needed for all three plasma pulse phases, namely (i) plasma start-up, (ii) plasma flattop and (iii) pulse termination and/or off-normal event shut-down. During the first phase the H\&CD power is used for the a) plasma breakdown, b) burn-through, c) assisted current ramp-up and d) heating to H-mode, while during the second phase it is used for e) burn control, f) MHD control, g) impurity control and depending on the regime more or less $h$ ) current drive. During the third phase it serves for the i) assisted rampdown. No H\&CD functionality is planned for dwell time between pulses, but if so, H\&CD methods could be used for T-desorption, wall conditioning, etc. Depending on further physics findings, the optimal H\&CD drive mix can be determined, meanwhile for the present DEMO the assumptions are to use (i) EC for assisted breakdown, electron heating, CD and MHD control, (ii) NB for heating and CD, (iii) IC for mainly bulk ion heating, impurity control and it could potentially contribute to CD. Studies done so far suggest LH is not considered for the pulsed machine but might play a significant role for an efficient far off-axis CD in the steady-state regime.

\subsubsection{Auxiliary Heating Power}

The thermal power balance in a tokamak is given by $P_{\text {fusion }}+P_{\text {aux }}=P_{\text {loss }}+P_{\text {neutron }}$ with $P_{\text {fusion }}=P_{\text {neutron }}+P_{\alpha}$. and $P_{\text {loss }}=P_{\text {rad }}+P_{\text {sep }}$, where the radiation losses inside the separatrix $P_{\text {rad }}$ include line radiation, Bremsstrahlung and synchrotron radiation. The power crossing the separatrix $P_{\text {sep }}$ determines the thermal loads on the divertor. For the DEMO reference case it was assumed $P_{\text {sep }} \approx 1.3 \cdot P_{L-H}$, (present studies use $P_{\text {sep }} \approx P_{L-H}$ ) where $P_{L-H}$ is the H-mode threshold. The H\&CD power of 50MW had initially been fixed in the PROCESS code runs, strongly influenced by ITER studies and requires re-assessment. In fact first rough calculations indicate the required power for the ramp-up to be at least 50MW and possibly even beyond 100MW, depending on the assumptions and tokamak start-up time, whereby a longer ramp-up time requires lower H\&CD power.

\subsubsection{Current Drive Power}

The CD power needed depends on several plasma parameters and systems properties, but also on geometrical factors: in case of NB the tangential radii of the beams and in case of EC the launching angles and positions of power deposition of the waves, the latter are also the case for IC. Several studies were made to optimize the CD efficiencies assuming either EC, NB or IC []]-[ㅁ]. Not surprisingly, the CD efficiency depends on the plasma profiles. The profiles were calculated beginning from 0D-code PROCESS to optimize the tokamak. The outcome of those studies was then fed into the transport codes ASTRA/TRANSP yielding the 1D kinetic plasma profiles. In all cases of the reference design (with or w/o CD) and the aspect ratio scans, the bootstrap current has been kept at a realistic level of about 35\%.

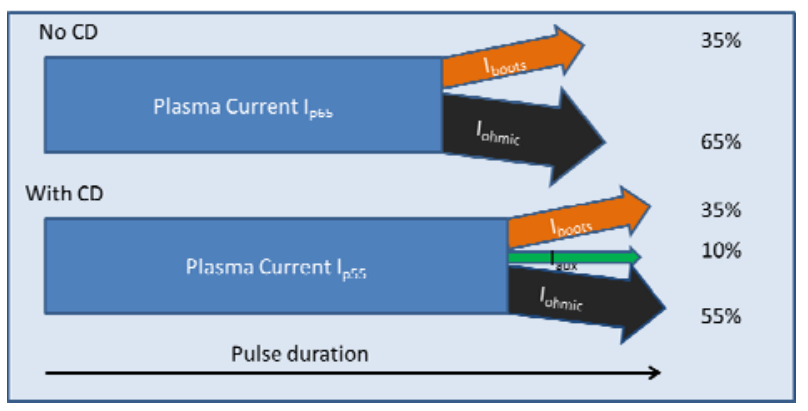

Fig. 1. Diagram of the pulse duration and plasma current with / without CD.

The plasma burn time is given by $t_{\text {burn }} \sim\left(\left|\Phi_{\text {tot }}\right|-\Phi_{\text {start }}\right) /\left(I_{p} \cdot R_{p} \cdot\left\{1-f_{\text {non-inductive }}\right\}\right)$.

$\Phi_{\text {tot }}$ is the total magnetic flux of the central solenoid coil, $\Phi_{\text {start }}$ is the flux required for the start-up of the plasma and $R_{p}$ is the plasma resistance. By assuming $\Phi_{\text {tot }}, \Phi_{\text {start }}$ and $R_{p}$ are constant one can calculate the plasma pulse length with versus w/o CD. Applying $50 \mathrm{MW}$ of auxiliary power for CD fraction $f_{a u x, C D}$ the total plasma current $I_{P}$ driven from $f_{a u x, C D}$ is about $10 \%$ (depending on the CD efficiency). The noninductive fraction is $f_{\text {non-inductive }}=f_{\text {aux }, C D}+f_{B S}$, were $f_{B S}$ stands for the bootstrap-fraction. The calculated pulse length with CD slightly increases compared to the 
pure inductively driven pulse by about $25 \mathrm{~min}$ to $\sim 2.7 \mathrm{~h}$, cf. Fig. 1.

\subsubsection{EC assisted breakdown and MHD control}

The results of first simulations for the pulse start show a modest power for the plasma breakdown and burn-through of about 6-12MW. Also the question of magnetohydrodynamics (MHD) control was considered during the assessments. The stabilisation of the Neoclassical Tearing Mode (NTM) is achieved by EC control. In a first scan for different poloidal and toroidal launch angles at the $q=2$ and $q=3 / 2$ surfaces the injected EC wave power was set to be $4.5-7 \mathrm{MW}$ and it could be shown that with this EC power the stabilisation criterion could be met over a wide radial range [20].

\section{H\&CD Systems}

The modularity of the systems very much influences their total availability and possible up-time. Whereas in case of EC the gyrotrons have about 1MW (@240GHz) per unit and in case of failure of one or more units could remain operational and guarantee an intrinsic high overall EC system reliability. The NB power of a single injector is rather high $(\sim 20-25 \mathrm{MW} @ 1 \mathrm{MeV})$. It is therefore foreseen to use modular NB sources inside the injector to increase the NB system overall availability in case of a fault in one source.

The physics studies for the different H\&CD systems were made assuming two types of kinetic profiles a) flat and b) peaked density case. They were selected to represent the boundaries in which a later profile is expected. The related peak temperatures are varying between $19 \mathrm{keV}$ and $26 \mathrm{keV}$ and the core density varies between $9 \cdot 10^{19} \mathrm{~m}^{-3}$ and $16 \cdot 10^{19} \mathrm{~m}^{-3}$. This has a major influence on the choice of technology and the injection point as can be seen below, cf. also [6][14].

\subsection{Negative Neutral Beam Injection (NNBI) system}

The NNBI system of DEMO shall benefit as much as possible from ITER. The specifications require a NB system with $1 \mathrm{MeV}$ and a total power of $>50 \mathrm{MW}$. The following DEMO relevant work has been carried out:

- Efficiency studies show that the CD efficiency is reduced by $<5 \%$ (on axis, peaked) to $10 \%$ (overall average) for the case of beam energy of $1 \mathrm{MeV}$ compared to $1.5 \mathrm{MeV}$, simulated by PENCIL code [14] for different tangential radii and elevations, combined with shinethrough studies. The studies also show that far off-axis CD increases the NB CD efficiency, cf. Fig. 2.

- Investigating Cs-reduced sources and non caesiated / alternative sources, e.g. matrix sources [10], negative ion production on boron-doped-diamond (BDD) [12], modelling of Cs chemistry and the plasma source.

- Improving RF-coupling and testing of recently commissioned solid state RF-generator for NB sources. - Increasing the wall plug efficiency, presently 24\%, by implementing a negative (and positive) ion energy recovery system to $29 \%$ (34\% respectively) [1] was studied and with small scale mock up tested.
- Shielding and integration studies running MCNP code were done to determine whether the TF coils would be adequately protected around the NB port.

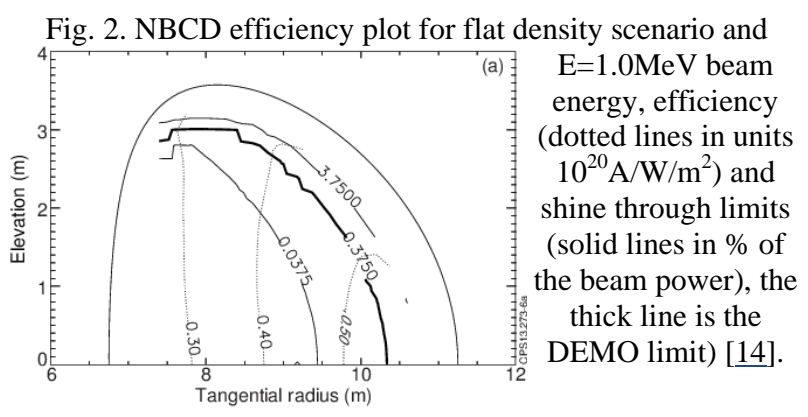

- Reducing the neutralisation losses by simulation to arrive at an optimized design of the beam neutralizer.

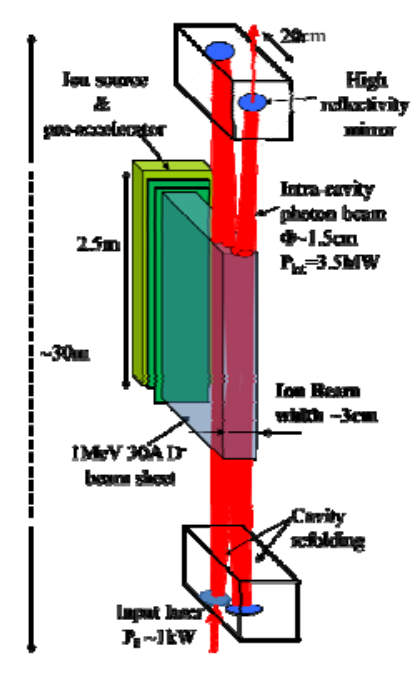

- Demonstrating the feasibility of photoneutralization [15], the high finesse cavity (including stability and erosion of mirrors) and the CW high power lasers was examined, see Fig. 3.

- High voltage breakdown behaviour was improved by modelling effects of depositions on electrodes and HV-bushings made of stainless steel.

Fig. 3. Schematic of the beam neutralization by a re-folded Fabry-Pérot cavity [ㅌ].

\subsection{EC system}

The selection of the operating frequency for EC is fundamental for development of the gyrotrons, transmission lines and launcher system. The optimum operating frequency for heating is given by the fundamental electron resonance frequency $f_{c, e}=\frac{\varpi_{c, e}}{2 \pi}=\frac{1}{2 \pi} \frac{e B}{m_{e}}=28 G H z \cdot B[T]$. For the DEMO reference design, shown in Table 1 , it results in heating frequency of about 190GHz(@6.8T). To find the optimum EC current drive (ECCD) frequency simulations were carried out in []]. Different injection points (equatorial and top launch) and injection angles have been assumed. The optimum ECCD frequency has been found to be $280 \mathrm{GHz}$ using top launch. A reduction in frequency to relax the $R \& D$ efforts, taking into consideration e.g. the required magnetic field of the gyrotron superconducting (SC) magnet and the operation at very high order modes, leading to a good compromise, where the frequency might be $\sim 240 \mathrm{GHz}$ with tolerable drawbacks ( 15-20\%) in CD efficiency.

Considering a future-multipurpose multi-frequency gyrotron, the following operating frequencies were identified: $136 \mathrm{GHz}$ (cf. W7X 140GHz), $170 \mathrm{GHz}$ (ITER), 204GHz, 238GHz (envisaged for CD for the present DEMO reference design) and eventually possible 
272GHz. A frequency of $272 \mathrm{GHz}$ requires a very high magnetic field of the SC magnet of about 12T, whereas for $238 \mathrm{GHz}$ it is only $10 \mathrm{~T}$. The assumed output power per unit for $238 \mathrm{GHz}$ will be above $1 \mathrm{MW}$, whereas for $272 \mathrm{GHz}$ it will below $1 \mathrm{MW}$.

Future gyrotron R\&D has to concentrate on a sufficient output power level at operating frequencies above $200 \mathrm{GHz}$, plug-in efficiencies above $60 \%$, multipurpose operation, frequency step-tunability, long lifetime operation at CW operation and very high repetition rates. Gyrotron technologies developed for ITER are good starting point for DEMO. Nevertheless to achieve DEMO targets, key components like the electron emission and electron optical system has to be improved. Additionally broadband window technologies, e.g. chemical vapour deposition diamond (CVD) Brewsterangle windows [17] and multi-stage depressed collectors will have to be developed (Table 2).

Table 2. Broadband Window Technologies for DEMO

\begin{tabular}{l|c|c}
$\begin{array}{c}\text { The X stands for the envisaged options } \\
\text { under assessment }\end{array}$ & $\begin{array}{l}\text { Gyrotron } \\
\text { Window }\end{array}$ & $\begin{array}{l}\text { Torus } \\
\text { Window }\end{array}$ \\
\hline Brewster Angle Window & $\mathrm{X}$ & $\mathrm{X}$ \\
\hline Double Disc Window & $\mathrm{X}$ & $\mathrm{X}$ \\
\hline Grooved Diamond Disk Window & $\mathrm{X}$ & \\
\hline Ring Resonator Based Window & $\mathrm{X}$ &
\end{tabular}

As part of the launcher studies different configurations on the remote steering (avoiding movable parts and mirrors due to high neutron and radiation fluxes) and their focussing capabilities of the EC millimetre wave were assessed for equatorial launchers and are being pursued for the upper port launcher. Integration studies showed that some space in the DEMO upper port is available but tight mainly due to the necessary integration of the blanket cooling pipes [18].

\subsection{IC system}

In contrast to the very high EC frequency in the $\mathrm{GHz}$ range, the wave frequencies of a few tens of $\mathrm{MHz}$ are typically used for IC heating. The resonant frequencies for the central IC heating can be determined from $f_{c, i}=15.2 \mathrm{MHz} \cdot(N \cdot Z / A) \cdot B[T]$, where $N$ is the heating harmonics number, and $Z / A$ is the charge-tomass ratio of the resonant ions. For a DEMO operating at 6.8T, IC heating of tritium ions at the second harmonics requires the wave frequency of $\sim 70 \mathrm{MHz}$.

To optimize the CD efficiency, various design options were considered. The optimal frequency is in the $100-350 \mathrm{MHz}$ range for top launch with high harmonics fast wave $\mathrm{CD}$ and is $25-100 \mathrm{MHz}$ for fast wave CD (FWCD) for equatorial launch. These work results refer to an ITER-like antenna implemented in a port []][9]].

Recently the concept of a distributed antenna [19] for the WP2014-2020 was taken as a very promising design choice for DEMO, see Fig. 4, having several advantages:

- Easy remote handling of the passive antenna structure as integrated part of the blanket segments.

- No extra cooling required, shared blanket cooling.
- Significantly reduced sheath-effects, sputtering and arcing or similar problems by reduced power density and voltage.

- Positioning of the antenna in the upper region avoids space occupations of ports with positive impact on the Tritium Breeding Ratio (TBR). Such a wave launcher location also results in higher FWCD efficiency [9].

The conceptual design of the distributed antenna is in progress, further simulations and small scale testing are planned in the coming years to underpin the IC antenna strategy. An important aspect will be the proper material-selection and cooling of the plasma-facing front part of the antenna.

Fig. 4. One sector of DEMO with an in-blanket IC antenna.

\subsection{LH System}

Comprehensive work was done for the further investigation on the LH systems, in particular on (i) LH physics using ASTRA and FRTC codes, (ii) detailed work on main transmission lines and components to optimize converters, mode filters and bends, (iii) on CVD RF windows, on (iv) LH launchers, and (v) on RAMI. For the high power and long pulse (or CW) DEMO operation a slotted waveguide antenna (SWA) instead of presently used antenna types as Grill-, Multijunction- or PAMantennas was proposed. The integration and first low power tests of a SWA LH launcher at $5 \mathrm{GHz}$ will be performed on the COMPASS tokamak in Prague.

Fig. 5. Narrow side SWA port integration in COMPASS.

\section{Acknowledgments}

This work has been carried out within the framework of the EUROfusion Consortium and has received funding from the European Union's Horizon 2020 research and innovation programme under grant agreement number 633053. The views and opinions expressed herein do not necessarily reflect those of the European Commission.

\section{References}

[1] M. Q. Tran, this conference (I4.3).

[2] F. Romanelli et al. EFDA Fusion roadmap.

[3] H. Zohm et al. Nucl. Fusion 53 (2013) 073019.

[4] H. Zohm Fusion Sci. Technol. 58 (2010), 613-624.

[5] R. Wenninger et al. IAEA (2014) (submitted for publication).

[6] E. Poli, F. Romanelli, P. Barabaschi, D. Borba, G. Federici, L. Horton, et al. 2013 Nucl. Fusion 53 (2013) 013011.

[7] Surrey E., A. Benn, I. Jenkins, M. Porton, W. Timmis, A. Turner, et al. Fusion Eng. Des. 87 (2012) 373-383.

[8] R. Koch, E. Lerche, D. Van Eester, M. Nightingale, AIP Conference Proceedings vol. 1406 (2011), 349-352.

[9] E. Lerche, D. Van Eester, A. Messian, T. Franke, EFDAPPPT contributors, AIP Conference Proceedings vol. 1580 (2014) 338-341. 
[10] R. Kemp, M. Nakamura, D. J. Ward, K. Tobita, G. Federici, 24th IAEA Fusion Energy Conference (2012).

[11] St. Lishev, Ts. Paunska, A. Shivarova, Kh. Tarnev, Rev. Sci. Instrum. 83 (2012), 02A702, 1-5.

[12] P. Kumar, A. Ahmad, C. Pardanaud, M. Carrère, J. M. Layet, G. Cartry,. et al. Appl. Phys. 44 (2011) 372002, 4pp.

[13] R. Mc Adams, Rev. Sci. Instrum. 85 (2014) $02 B 319$.

[14] I. Jenkins, D. L. Keeling, E. Surrey, $25^{\text {th }}$ SOFE Conference (2013).

[15] A. Simonin, AIP Conference Proceedings vol. 1390 (2011) 494-504.

[16] A. Simonin, H. P. L. de Esch, P. Garibaldi, C. Grand, S. Bechu, A. Bès, et al., $4^{\text {th }}$ International Symposium on Negative Ions, Beams and Sources (NIBS) (2014) AIP Conference Proceedings 2015 (to be published).

[17] G. Gantenbein, IEEE Trans. Electron 61 (2014) 1806-1811.

[18] C. Bachmann, this conference (P1.124).

[19] G. Bosia, AIP Conference Proceedings vol. 1406 (2011) 133-136.

[20] Final reports DAS-HCD-WP2013 EFDA_D_2M7L5P. 